\title{
Copper cyanide polymers - new directions
}

Peter W. R. Corfielda, Leena N. Rachidb, Daniel J. Garciac and Alison N. McCostis ${ }^{d}$

a Chemistry Department, Fordham University, 441 E. Fordham Road, Bronx, NY 10458, pcorfieldefordham.edu

b Chemistry Department, Fordham University, 441 E. Fordham Road, Bronx, NY 10458, lrachidefordham.edu

c Chemistry Department, Fordham University, 441 E. Fordham Road, Bronx, NY 10458, dgarcia58efordham.edu

d Chemistry Department, Fordham University, 441 E. Fordham Road, Bronx, NY 10458, amccostisefordham.edu

Our original goal was the designed synthesis and X-ray structure determination of neutral mixed-valence copper cyanide polymers with $\mathrm{Cu}^{\mathrm{II}}$ complexed with amine bases, and a $\mathrm{Cu}^{\mathrm{I}} \mathrm{Cu}^{\mathrm{II}} \mathrm{CN}$ framework. We determined the X-ray structures of a number of 1D, 2D and 3D mixed valence polymers of this type. Some syntheses, however, led to unexpected products that could not contain $\mathrm{Cu}^{\text {II }}$ as they were colorless. X-ray structural analyses indicated anionic 2D or 3D CuI networks with guest cations, and suggested further systematic study. By altering reaction conditions to produce intentionally more compounds of this type, we have characterized a number of new polymeric anionic $\mathrm{Cu}^{\mathrm{I}} \mathrm{CN}$ structures, with charge neutrality obtained by various protonated guest amines and diamines. For instance, whereas crystallization of $\mathrm{Cu}(\mathrm{CN}) \mathrm{x}^{1-x}$ mixtures in the presence of $\mathrm{N}$-ethylethylenediamine (eten) affords large blue crystals of $\mathrm{Cu}_{2}(\mathrm{CN})_{3}$ eten 2 via air-oxidation, at

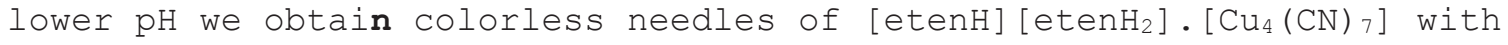
the anionic CuCN framework shown. We will present the new structures and discuss how different bases can drive formation of different topologies for the CuCN frameworks.

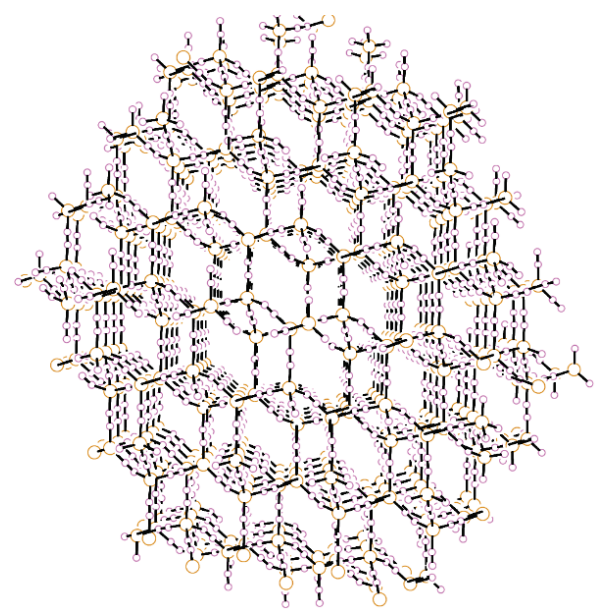

